\title{
Advances in the Statistical Characterization of Fading: From 2005 to Present
}

\author{
José F. Paris \\ Departamento Ingeniería de Comunicaciones, Universidad de Málaga, 29071 Málaga, Spain \\ Correspondence should be addressed to José F. Paris; paris@ic.uma.es
}

Received 9 May 2014; Accepted 23 June 2014; Published 10 July 2014

Academic Editor: Paschalis Sofotasios

Copyright (C) 2014 José F. Paris. This is an open access article distributed under the Creative Commons Attribution License, which permits unrestricted use, distribution, and reproduction in any medium, provided the original work is properly cited.

This review paper presents some of the most significant advances in statistical characterization of wireless communication fading. We will focus on the fundamental statistical characterizations of the fading distributions in terms of the probability density function (PDF), the cumulative density function (CDF), and the moment generating function (MGF). The period from 2005 to present will be considered in order to complement the information which can be found in the specialized literature like Simon and Alouini's book on digital communications over fading channels. We present both the advances on classical fading models and a brief summary of some selected new fading models. Some open problems in the field will be also stated.

\section{Introduction}

Fading is an important peculiarity of wireless communication channels. Statistical characterization of fading is a key aspect for the analysis and simulation of wireless communication systems. In the last years, there has been a huge amount of research work on statistical characterization of wireless communication fading. The most comprehensive account of this research topic is Simon and Alouini's book on digital communications over fading channels [1]. This book presents the statistical characterization of the most popular fading models by 2005 .

In this review paper, in order to complement the information available in [1], a selection of the new results on fading statistics in the period from 2005 to present will be considered. Only single-input single-output (SISO) flat fading channels are considered; for an introduction to multipleinput multiple-output (MIMO) fading channels the reader should consider $[2,3]$. We will focus on the fundamental statistical characterizations of the fading distributions in terms of the probability density function (PDF), the cumulative density function (CDF), and the moment generating function (MGF). We will also pay special attention to the exact closedform results concerning the PDF, CDF, and MGF of the fading distributions. The advances on classical fading models and some results on new fading models will be presented.
Along the paper, some open problems on fading statistics will be stated.

The remainder of this paper is organized as follows. In Section 2 the precise mathematical definitions of the fundamental statistics are provided. The advances concerning the classical fading distributions are presented in Section 3 and some selected results on new fading models are presented in Section 4. Finally, some conclusions are given in Section 5.

\section{Statistical Preliminaries}

Let us denote $R$ which is the envelope of the fading channel. In this review paper we will focus on the fundamental statistics of the instantaneous signal-to-noise ratio (SNR) $\gamma$. Let $E[\cdot]$ be the expectation operator. The instantaneous SNR can be expressed in terms of the channel envelope $R$ and the average $\mathrm{SNR} \bar{\gamma} \triangleq E[\gamma]$ :

$$
\gamma=\frac{R^{2}}{E\left[R^{2}\right]} \bar{\gamma}
$$

The CDF of the instantaneous SNR $\gamma$ can be obtained from the $\operatorname{PDF} f_{\gamma}(\gamma)$ as follows:

$$
F_{\gamma}(\gamma)=\int_{0}^{\gamma} f_{\gamma}(\xi) d \xi
$$


while the MGF is computed as

$$
\mathscr{M}_{\gamma}(s)=E\left[e^{s \gamma}\right]=\int_{0}^{\infty} f_{\gamma}(\xi) e^{s \xi} d \xi
$$

Note that the definition of the MGF given in (3) differs from the unilateral Laplace transform definition in the sign of the variable $s$.

From a pure statistical point of view, the three characterizations of the instantaneous SNR $\gamma$ (the PDF, the CDF, and the MGF) are equivalent; however, from the point of view of the performance analysis of fading channels, the MGF and the CDF play an important role in the computation of key performance metrics such as error probability, outage probability, or outage capacity [1].

\section{Advances on Classical Fading Models}

In this section we will consider as classical fading models the following: Rayleigh, Nakagami- $q$ (Hoyt), Nakagami- $n$ (Rice), Nakagami- $m$, Weibull, Beckmann, Lognormal, $K$, and Rician shadowed. All these fading models are well described in [1].

The PDF, CDF, and MGF of the Rayleigh, Nakagami- $m$, Nakagami- $n$ (Rice), and Weibull distributions are expressed in closed-form, and the corresponding expressions are found in [1].

Only the PDF and MGF of the Nakagami-q (Hoyt) distribution are given in [1]. A closed-form expression for its CDF was derived in $2009[4,5]$ :

$$
\begin{aligned}
F_{\gamma}(\gamma)= & Q\left(u(q) \sqrt{\frac{\bar{\gamma}}{\bar{\gamma}}}, v(q) \sqrt{\frac{\gamma}{\bar{\gamma}}}\right) \\
& -Q\left(v(q) \sqrt{\frac{\bar{\gamma}}{\gamma}}, u(q) \sqrt{\frac{\bar{\gamma}}{\bar{\gamma}}}\right), \quad \gamma \geq 0,
\end{aligned}
$$

where $\bar{\gamma} \triangleq E[\gamma], q$ is the Hoyt shape parameter, $u(q) \doteq\left(\sqrt{1-q^{4}} / 2 q\right) \sqrt{(1+q) /(1-q)}, v(q) \doteq\left(\sqrt{1-q^{4}} / 2 q\right)$ $\sqrt{(1-q) /(1+q)}$, and $Q(\cdot)$ is the Marcum Q function [1].

Interestingly, the Beckmann fading distribution includes the Rayleigh, Nakagami-q (Hoyt), Nakagami-n (Rice), and Nakagami- $m$ distributions as particular cases by a suitable combination of its parameters. Its MGF is expressed in elementary terms [1], but closed-form expressions for both its PDF and its CDF in terms of classical or well-studied special functions are challenging open problems.

Beyond channel modeling, the Lognormal distribution is widely used; its PDF and CDF are easily expressed in closed-form; however, no closed-form expression is known for its MGF. A recent paper on this particular problem which derives an approximate expression is [6].
The PDF and MGF of the $K$ distribution are expressed in closed-form in [1]. Its CDF was derived in closed-form in 2006 [7]:

$$
\begin{aligned}
F_{\gamma}(\gamma)= & \pi \csc (\pi(\nu-1)) \\
\times & {\left[\frac{(\nu \gamma / \bar{\gamma}){ }_{1} F_{2}(1 ; 2-\nu, 2 ; \nu(\gamma / \bar{\gamma}))}{\Gamma(\nu) \Gamma(2-\nu)}\right.} \\
& \left.\quad-\frac{(\nu \gamma / \bar{\gamma})_{1}^{\nu} F_{2}(\nu ; \nu, 1+\nu ; \nu(\gamma / \bar{\gamma}))}{\Gamma(\nu) \Gamma(1+\nu)}\right],
\end{aligned}
$$

where $\bar{\gamma} \triangleq E[\gamma], \nu$ is the distribution shaping parameter, $\Gamma(\cdot)$ is the gamma function, and ${ }_{p} F_{q}(\cdot)$ is the generalized hypergeometric function [8].

As in the previous model, the PDF and MGF of the Rician shadowed distribution are expressed in closed-form in [1]. Its CDF was derived in closed-form in 2010 [9]:

$$
\begin{aligned}
F_{\gamma}(\gamma) & \\
= & \frac{m^{m}(1+K)}{(m+K)^{m}} \frac{\gamma}{\bar{\gamma}} \\
& \times \Phi_{2}\left(1-m, m ; 2 ;-\frac{1+K}{\bar{\gamma}} \gamma,-\frac{1+K}{\bar{\gamma}} \frac{m \gamma}{K+m}\right),
\end{aligned}
$$

where $\bar{\gamma} \triangleq E[\gamma], K$ is the classical Rice parameter, $m$ is the parameter corresponding to the line-of-sight (LOS) fluctuations, and $\Phi_{2}(\cdot)$ is the bivariate confluent hypergeometric function [8].

\section{New Fading Models}

In this section the author has selected five new fading models which are not listed in [1]. The models presented in this section do not exhaust the list of new fading models recently proposed and not included in [1].

4.1. The $\eta-\mu$ Fading Model. This model was first proposed by Yacoub in 2000 [10] and later enhanced in [11]. From a physical point of view, this model corresponds to a nonline-of-sight (NLOS) scenario where different clusters of multipath waves can propagate simultaneously. The PDF of the $\eta-\mu$ distribution in format 1 (see [11] for the details) is given by

$$
\begin{gathered}
f_{\gamma}(\gamma)=\frac{2 \sqrt{\pi} \mu^{\mu+1 / 2} h^{\mu}}{\bar{\gamma} \Gamma(\mu) H^{\mu-1 / 2}}\left(\frac{\gamma}{\bar{\gamma}}\right)^{\mu-1 / 2} \\
\times \exp \left(-\frac{2 \mu h \gamma}{\bar{\gamma}}\right) I_{\mu-1 / 2}\left(\frac{2 \mu H \gamma}{\bar{\gamma}}\right), \\
h \triangleq \frac{2+\eta^{-1}+\eta}{4}, \\
H \triangleq \frac{\eta^{-1}-\eta}{4},
\end{gathered}
$$

where $\bar{\gamma} \triangleq E[\gamma], I_{p}(\cdot)$ is the modified Bessel function of the first kind [8], $\eta$ is a positive real number representing 
the scattered-wave power ratio between the in-phase and quadrature components of each cluster of multipath, and $\mu$ is a positive real number representing the effective number of propagation clusters. This model contains the Rayleigh, Nakagami-q (Hoyt), and Nakagami-m models as particular cases by a suitable combination of the parameters $\eta$ and $\mu$. In [11] a physical model was given for the $\eta-\mu$ model when $\mu$ is a natural number; this model is useful for simulation of $\eta$ $\mu$ random variables since these can be expressed in terms of Gaussian random variables. The MGF corresponding to the PDF given in (7) was expressed in elementary terms in 2008 [12]:

$$
\mathscr{M}_{\gamma}(s)=\left(\frac{4 \mu^{2} h}{(2(h-H) \mu+s \bar{\gamma})(2(h+H) \mu+s \bar{\gamma})}\right)^{\mu} .
$$

In 2010, an exact closed-form expression for the CDF of the $\eta$ $\mu$ distribution was derived in terms of the bivariate confluent function [13]:

$$
\begin{aligned}
F_{\gamma}(\gamma) & \\
= & \frac{\left(1-(H / h)^{2}\right)^{\mu}}{\Gamma(1+2 \mu)}\left(\frac{2 h \mu \gamma}{\bar{\gamma}}\right)^{2 \mu} \\
& \times \Phi_{2}\left(\mu, \mu ; 1+2 \mu ;-\left(1+\frac{H}{h}\right) \frac{2 h \mu \gamma}{\bar{\gamma}},-\left(1-\frac{H}{h}\right) \frac{2 h \mu \gamma}{\bar{\gamma}}\right) .
\end{aligned}
$$

Expressions (7), (8), and (9) complete the fundamental characterization of the $\eta-\mu$ fading model by closed-form expressions.

4.2. The $\kappa-\mu$ Fading Model. This model was also proposed by Yacoub in 2000 [14] and later enhanced in [11]. From a physical point of view, this model corresponds to a line-ofsight (LOS) scenario where different clusters of multipath waves can propagate simultaneously. The PDF of the $\kappa-\mu$ distribution is given by

$$
\begin{aligned}
f_{\gamma}(\gamma)= & \frac{\mu(1+\kappa)^{(\mu+1) / 2}}{\kappa^{(\mu-1) / 2} \bar{\gamma} \exp (\mu \kappa)}\left(\frac{\gamma}{\bar{\gamma}}\right)^{(\mu-1) / 2} \\
& \times \exp \left(-\frac{\mu(1+\kappa) \gamma}{\bar{\gamma}}\right) I_{\mu-1}\left(2 \mu \sqrt{\frac{\kappa(1+\kappa) \gamma}{\bar{\gamma}}}\right),
\end{aligned}
$$

where $\bar{\gamma} \triangleq E[\gamma], \kappa$ is a real positive number representing the power ratio between dominant and scattered-wave components, and $\mu$ is a positive real number representing the effective number of propagation clusters. This model contains the Rayleigh, Nakagami- $n$ (Rice), and Nakagami- $m$ models as particular cases by a suitable combination of the parameters $\kappa$ and $\mu$. In [11] a physical model was also given for the $\kappa$ $\mu$ model when $\mu$ is a natural number; this model is useful for simulation of $\kappa-\mu$ random variables since these can be expressed in terms of Gaussian random variables. The CDF of the $\kappa-\mu$ distribution was directly given in [11] in terms of the generalized Marcum $Q$ function [1]:

$$
F_{\gamma}(\gamma)=1-Q_{\mu}\left(\sqrt{2 \kappa \mu}, \sqrt{2(1+\kappa) \mu \frac{\gamma}{\bar{\gamma}}}\right)
$$

while the MGF was expressed in exact closed-form in 2008 [12]:

$$
\mathscr{M}_{\gamma}(s)=\left(\frac{\mu(1+\kappa)}{\mu(1+\kappa)+s \bar{\gamma}}\right)^{\mu} \exp \left(\frac{\mu^{2} \kappa(1+\kappa)}{\mu(1+\kappa)+s \bar{\gamma}}-\mu \kappa\right) .
$$

Therefore, expressions (10), (11), and (12) provide a complete set of alternative characterizations for the $\kappa-\mu$ fading model.

4.3. The $\alpha-\mu$ Fading Model. Again, this model was proposed by Yacoub in 2002 [15] and improved in 2007 [16]. From a physical point of view, this model is appropriate to consider nonlinear propagation phenomena. In fact, the $\alpha-\mu$ distribution is a rewritten form of the Stacy (generalized gamma) distribution. Defining $\Omega_{\alpha}=E\left[R^{\alpha}\right]$, the PDF of $\gamma=\left(R^{2} / \Omega_{2}\right) \bar{\gamma}$ in this model is given by

$$
f_{\gamma}(\gamma)=\frac{\alpha \mu^{\mu} \gamma^{(\alpha \mu / 2)-1}}{2 \Gamma(\mu) \tilde{\gamma}^{\alpha \mu / 2}} \exp \left(-\mu\left(\frac{\gamma}{\tilde{\gamma}}\right)^{\alpha / 2}\right)
$$

where $\bar{\gamma} \triangleq E[\gamma]$ and $\tilde{\gamma} \triangleq\left(\Omega_{\alpha}^{2 / \alpha} / \Omega_{2}\right) \bar{\gamma}$; otherwise, $\alpha$ and $\mu$ are shaping parameters. The CDF of the $\alpha-\mu$ fading model is expressed as

$$
F_{\gamma}(\gamma)=\frac{\Gamma\left(\mu, \mu(\gamma / \tilde{\gamma})^{\alpha / 2}\right)}{\Gamma(\mu)}
$$

where $\Gamma(\cdot, \cdot)$ is the incomplete gamma function [8]. The MGF of this distribution was derived in exact closed-form in 2009 (see [17]) by the Meijer $G$ function [8]:

$$
\begin{aligned}
\mathscr{M}_{\gamma}(s)= & \frac{\alpha \mu^{\mu}}{2 \Gamma(\mu) \tilde{\gamma}^{\alpha \mu / 2}} \frac{k^{1 / 2} l^{(\alpha \mu-1) / 2}}{(2 \pi)^{(l+k-2) / 2} s^{\alpha \mu / 2}} \\
& \times G_{l, k}^{k, l}\left(\left(\frac{\mu}{\tilde{\gamma}^{\alpha / 2}}\right)^{k} \frac{l^{l}}{s^{l} k^{k}} \mid \frac{i(l, 1-\alpha \mu / 2)}{i(k, 0)}\right),
\end{aligned}
$$

where $i(n, \xi)=\xi / n,(\xi+1) / n, \ldots,(\xi+n-1) / n$ and $\alpha / 2=$ $l / k$ such that $\operatorname{gcd}(l, k)=1$ (great common divisor) in order to include noninteger values of $\alpha$. As happens with the two previous models, the PDF, CDF, and MGF of the $\alpha-\mu$ distribution are known in exact closed-form by expressions (13), (14), and (15).

4.4. The $\mathscr{G}$ Fading Model. The $\mathscr{G}$ distribution was first proposed by Frery et al. [18], in the context of synthetic aperture radar (SAR) image modeling. In 2008 Laourine et al. proposed the application of this model to wireless fading channels [19]. The $\mathscr{G}$ distribution can be seen as a composite 
Nakagami- $m$-inverse Gaussian distribution. The PDF of the $\mathscr{G}$ distribution is expressed in closed-form as [19]

$$
\begin{gathered}
f_{\gamma}(\gamma)=A \frac{\gamma^{m-1}}{(\sqrt{\alpha+\beta \gamma})^{m+1 / 2}} K_{m+1 / 2}(b \sqrt{\alpha+\beta \gamma}), \\
A \triangleq \frac{(\lambda \bar{\gamma})^{(1+2 m) / 4}}{\Gamma(m)} \sqrt{\frac{2 \lambda}{\pi \theta}} \exp \left(\frac{\lambda}{\theta}\right)\left(\frac{m}{\bar{\gamma}}\right)^{m}, \\
b \triangleq \frac{1}{\theta} \sqrt{\frac{\lambda}{\bar{\gamma}}} \\
\alpha \triangleq \lambda \bar{\gamma}, \quad \beta \triangleq 2 m \theta
\end{gathered}
$$

where $\bar{\gamma} \triangleq E[\gamma], K_{p}(\cdot)$ is the modified Bessel function of the second kind [8], the parameter $m$ comes from the underlying Nakagami- $m$ distribution, and the parameters $\lambda$ and $\theta$ correspond to the parameters of the underlying inverse Gaussian distribution. The CDF was also derived in [19]:

$$
\begin{aligned}
F_{\gamma}(\gamma) & \\
= & 1-A \Gamma(m) \\
& \times \sum_{k=1}^{m} \frac{2^{k} \gamma^{m-k}}{(\beta b)^{k}(m-k) !} \frac{K_{m-k+1 / 2}(b \sqrt{\alpha+\beta \gamma})}{(\sqrt{\alpha+\beta \gamma})^{m-k+1 / 2}},
\end{aligned}
$$

where $m$ is restricted to be a natural number. Otherwise, the MGF is given by [19]

$$
\begin{aligned}
\mathscr{M}_{\gamma}(s)= & +m \sum_{k=1}^{m-1} \frac{(-1)^{k+1} C_{m-1}^{k}}{(k+1) !} \\
& \times \sum_{p=0}^{k} C_{k}^{p}\left(2 \sqrt{\frac{\alpha s}{\beta}}\right)^{k+1-p} \Gamma(k+p+1) \\
& \times H_{-(k+p+1)}\left(\frac{b}{2} \sqrt{\frac{\beta}{s}}+\sqrt{\frac{s \alpha}{\beta}}\right),
\end{aligned}
$$

where $C_{p}^{k}$ is the binomial coefficient and $H_{p}(\cdot)$ is the Hermite function [8]. To the best knowledge of the author, finding exact closed-form expressions for the CDF and MGF of the $\mathscr{G}$ distribution, for arbitrary values of $m$, is an open problem.

4.5. The $\kappa-\mu$ Shadowed Fading Model. This model has been proposed by the author in 2014 [20]. It is a generalization of both the Rician shadowed model and the $\kappa$ - $\mu$ model. The PDF of the $\kappa-\mu$ shadowed distribution is given by

$$
\begin{aligned}
f_{\gamma}(\gamma)= & \frac{\mu^{\mu} m^{m}(1+\kappa)^{\mu}}{\Gamma(\mu)(m+\mu \kappa)^{m}}\left(\frac{\gamma}{\bar{\gamma}}\right)^{\mu-1} \\
& \times e^{-\mu(1+\kappa) \gamma / \bar{\gamma}}{ }_{1} F_{1}\left(m, \mu ; \frac{\mu^{2} \kappa(1+\kappa)}{\mu \kappa+m} \frac{\gamma}{\bar{\gamma}}\right),
\end{aligned}
$$

where $\bar{\gamma} \triangleq E[\gamma],{ }_{1} F_{1}(\cdot)$ is the confluent hypergeometric function [8], $\kappa$ is a real positive number representing the power ratio between dominant and scattered-wave components, $\mu$ is a positive real number representing the effective number of propagation clusters, and $m$ is a parameter associated with the LOS fluctuation. This model contains the Rician shadowed when $\mu=1$ and the $\kappa-\mu$ when $m \rightarrow \infty$. In [20] a physical model was also given for the $\kappa-\mu$ shadowed model when $\mu$ is a natural number; this model is useful for simulation of $\kappa-\mu$ shadowed random variables since these can be expressed in terms of Gaussian and gamma random variables.

The shaping parameters of this distribution have a clear influence on the amount of fading (AoF) of $\gamma$ [1]. In absence of LOS fluctuation $(m \rightarrow \infty)$ the AoF is due to multipath and depends on $\kappa$ and $\mu$ as follows [11]:

$$
\operatorname{AoF}[\gamma]=\frac{V[\gamma]}{E[\gamma]^{2}}=\frac{1}{\mu} \frac{1+2 \kappa}{(1+\kappa)^{2}},
$$

where $V[\cdot]$ is the variance operator. Note that the AoF in (20) is inversely proportional to $\mu$. Otherwise, the AoF of the LOS component is inversely proportional to $m$.

The CDF of the $\kappa-\mu$ shadowed distribution was also given in [20] in terms of the bivariate confluent hypergeometric function:

$$
\begin{aligned}
F_{\gamma}(\gamma)= & \frac{\mu^{\mu-1} m^{m}(1+\kappa)^{\mu}}{\Gamma(\mu)(m+\mu \kappa)^{m}}\left(\frac{\gamma}{\bar{\gamma}}\right)^{\mu} \\
& \times \Phi_{2}\left(\mu-m, m ; \mu+1 ;-\frac{\mu(1+\kappa)}{\bar{\gamma}} \gamma,\right. \\
& \left.-\frac{\mu(1+\kappa)}{\bar{\gamma}} \frac{m \gamma}{\mu \kappa+m}\right),
\end{aligned}
$$

while the MGF is expressed as [20]

$$
\begin{aligned}
\mathscr{M}_{\gamma}(s)= & \frac{(-\mu)^{\mu} m^{m}(1+\kappa)^{\mu}}{\bar{\gamma}^{\mu}(m+\mu \kappa)^{m}} \\
& \times \frac{(s-\mu(1+\kappa) / \bar{\gamma})^{m-\mu}}{(s-(\mu(1+\kappa) / \bar{\gamma})(m /(\mu \kappa+m)))^{m}} .
\end{aligned}
$$

Therefore, expressions (19), (21), and (22) provide a complete set of alternative characterizations for the $\kappa-\mu$ shadowed fading model.

\section{Conclusions}

This review paper presents some important results concerning fading statistics from 2005 to present. The new results on the most relevant classical fading distributions have been described. A selection of novel proposals for fading distributions is also included. A summary of relevant features of these novel distributions is shown in Table 1. Several open problems concerning the fading distributions have been also stated.

\section{Conflict of Interests}

The author declares that there is no conflict of interests regarding the publication of this paper. 
TABLE 1: Relevant statistical information on the novel fading distributions.

\begin{tabular}{|c|c|}
\hline Fading distribu & \\
\hline & The PDF, CDF, and MGF are expressed in closed-form (see [11-13]). \\
\hline$\eta-\mu$ & It does include the one-sided Gaussian, Hoyt, Rayleigh, and Nakagami- $m$ distributions as particular cases. \\
\hline & Specifically, it does not include the Rician, Weibull, and Lognormal distributions. \\
\hline & The PDF, CDF, and MGF are expressed in closed-form (see $[11,12])$ \\
\hline$\kappa-\mu$ & It does include the one-sided Gaussian, Rayleigh, Rician, and Nakagami- $m$ distributions as particular cases. \\
\hline & Specifically, it does not include the Hoyt, Weibull, and Lognormal distributions. \\
\hline & The PDF, CDF, and MGF are expressed in closed-form (see $[16,17])$ \\
\hline$\alpha-\mu$ & $\begin{array}{l}\text { It does include the one-sided Gaussian, Rayleigh, Nakagami- } m \text {, exponential, gamma, and Weibull distributions } \\
\text { as particular cases. }\end{array}$ \\
\hline & Specifically, it does not include the Hoyt, Rician, and Lognormal distributions. \\
\hline & $\begin{array}{l}\text { The PDF is expressed in closed-form (see [19]). The CDF and MGF are expressed in closed-form only when the } \\
\text { parameter } m \text { is a natural number (see [19]). }\end{array}$ \\
\hline$G$ & $\begin{array}{l}\text { It does include the one-sided Gaussian, Rayleigh, Nakagami- } m \text {, and inverse Gaussian distributions as particular } \\
\text { cases. }\end{array}$ \\
\hline & Specifically, it does not include the Hoyt, Rician, Weibull, and Lognormal distributions. \\
\hline & The PDF, CDF, and MGF are expressed in closed-form (see [20]). \\
\hline$\kappa-\mu$ shadowed & $\begin{array}{l}\text { It does include the one-sided Gaussian, Rayleigh, Nakagami- } m \text {, Rician, Rician shadowed, and } \kappa-\mu \text { distributions } \\
\text { as particular cases. }\end{array}$ \\
\hline & Specifically, it does not include the Hoyt, Weibull, and Lognormal distributions. \\
\hline
\end{tabular}

\section{Acknowledgments}

This work is partially supported by the Spanish Government and FEDER under Project TEC2011-25473.

\section{References}

[1] M. K. Simon and M-S. Alouini, Digital Communications over Fading Channels, John Wiley \& Sons, 2nd edition, 2005.

[2] I. E. Telatar, "Capacity of multiantenna Gaussian channels," European Transactions on Telecommunications, vol. 10, no. 6, 1999.

[3] M. Kang and M. Alouini, "Capacity of MIMO Rician channels," IEEE Transactions on Wireless Communications, vol. 5, no. 1, pp. 112-122, 2006.

[4] J. F. Paris, "Nakagami-q (Hoyt) distribution function with applications," Electronics Letters, vol. 45, no. 4, pp. 210-211, 2009.

[5] J. F. Paris, "Nakagami- $q$ (Hoyt) distribution function with applications," Electronics Letters, vol. 45, no. 8, pp. 210-211, 2009.

[6] S. Asmussen, J. L. Jensen, and L. Rojas-Nandayapa, "On the Laplace transform of the Lognormal distribution," Thiele Centre, 2013, http://math.au.dk/publs?publid=994.

[7] P. S. Bithas, N. C. Sagias, P. T. Mathiopoulos, G. K. Karagiannidis, and A. A. Rontogiannis, "On the performance analysis of digital communications over Generalized-K fading channels," IEEE Communications Letters, vol. 10, no. 5, pp. 353-355, 2006.

[8] I. S. Gradshteyn and I. M. Ryzhik, Table of Integrals, Series and Products, Academic Press, 7th edition, 2007.

[9] J. F. Paris, "Closed-form expressions for Rician shadowed cumulative distribution function," Electronics Letters, vol. 46, no. 13, pp. 952-953, 2010.

[10] M. D. Yacoub, “The $\eta-\mu$ distribution: a general fading distribution," in Proceedings of the 52nd IEEE Vehicular Technology Conference, vol. 2, pp. 872-877, Boston, Mass, USA, September 2000 .
[11] M. D. Yacoub, "The $\kappa-\mu$ distribution and the $\eta-\mu$ distribution," IEEE Antennas and Propagation Magazine, vol. 49, no. 1, pp. 6881, 2007.

[12] N. Y. Ermolova, "Moment generating functions of the generalized $\eta-\mu$ and $\kappa-\mu$ distributions and their applications to performance evaluations of communication systems," IEEE Communications Letters, vol. 12, no. 7, pp. 502-504, 2008.

[13] D. Morales-Jiménez and J.F. Paris, "Outage probability analysis for $\eta-\mu$ fading channels," IEEE Communications Letters, vol. 14, no. 6, pp. 521-523, 2010.

[14] M. D. Yacoub, "The $\kappa-\mu$ distribution: a general fading distribution," in IEEE VTS 54th Vehicular Technology Conference VTC Fall, vol. 3, pp. 1427-1431, Atlantic City, NJ, USA, October 2001.

[15] M. D. Yacoub, "The $\alpha-\mu$ distribution: a general fading distribution," in Proceedings of the 13th IEEE International Symposium on Personal, Indoor and Mobile Radio Communications (PIMRC '02), vol. 2, p. 629633, 2002.

[16] M. D. Yacoub, “The $\alpha-\mu$ distribution: a physical fading model for the Stacy distribution," IEEE Transactions on Vehicular Technology, vol. 56, no. 1, pp. 27-34, 2007.

[17] A. M. Magableh and M. M. Matalgah, "Moment generating function of the generalized $\alpha-\mu$ distribution with applications," IEEE Communications Letters, vol. 13, no. 6, pp. 411-413, 2009.

[18] A. C. Frery, H. Müller, C. D. C. F. Yanasse, and S. J. S. Sant'Anna, "A model for extremely heterogeneous clutter," IEEE Transactions on Geoscience and Remote Sensing, vol. 35, no. 3, pp. 648-659, 1997.

[19] A. Laourine, M.-S. Alouini, S. Affes, and A. Stéphenne, "On the performance analysis of composite multipath/shadowing channels using the G-distribution," in Proceedings of the IEEE International Conference on Communications (ICC '08), pp. 1333-1338, Beijing, China, May 2008.

[20] J. F. Paris, "Statistical characterization of $\kappa-\mu$ shadowed fading," IEEE Transactions on Vehicular Technology, vol. 63, no. 2, pp. 518-526, 2014. 

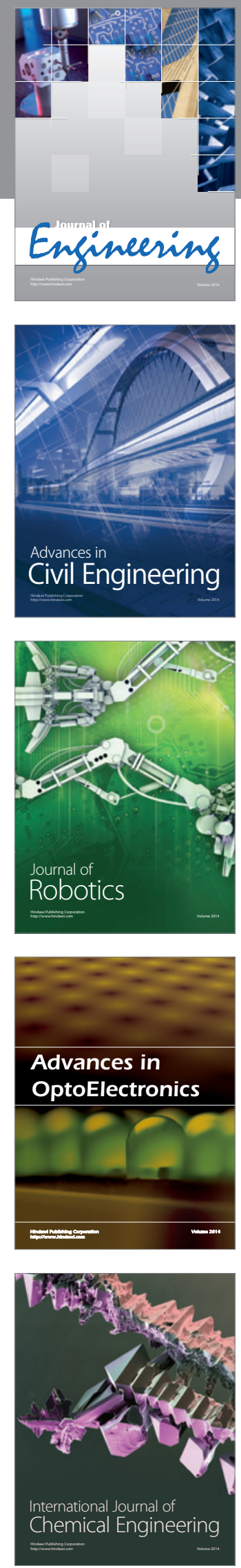

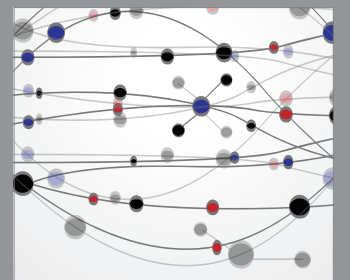

The Scientific World Journal
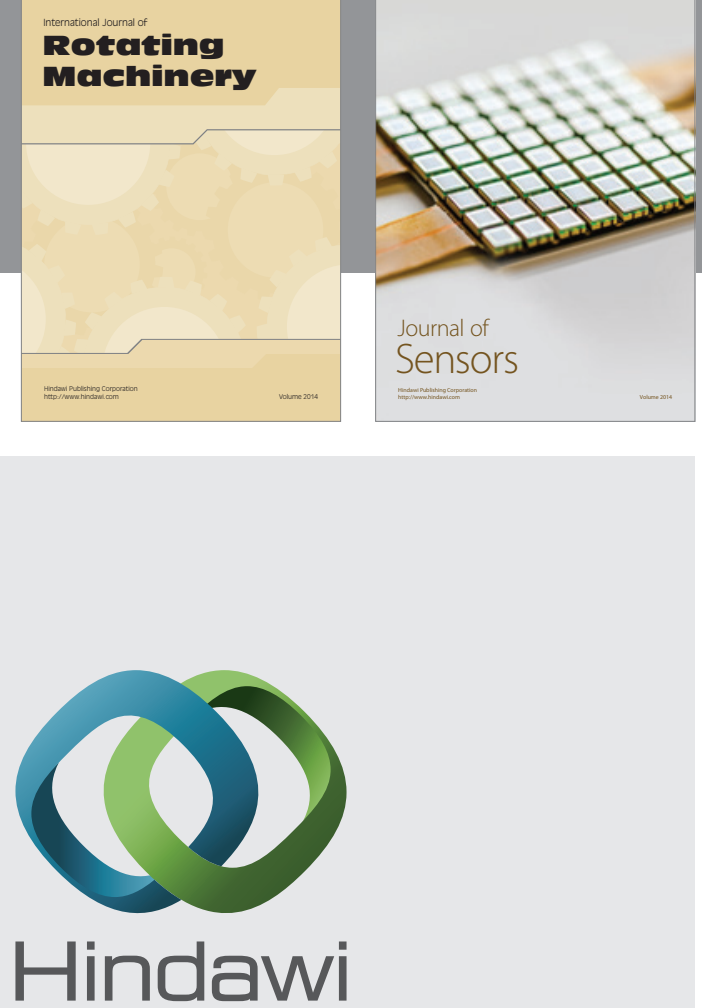

Submit your manuscripts at http://www.hindawi.com
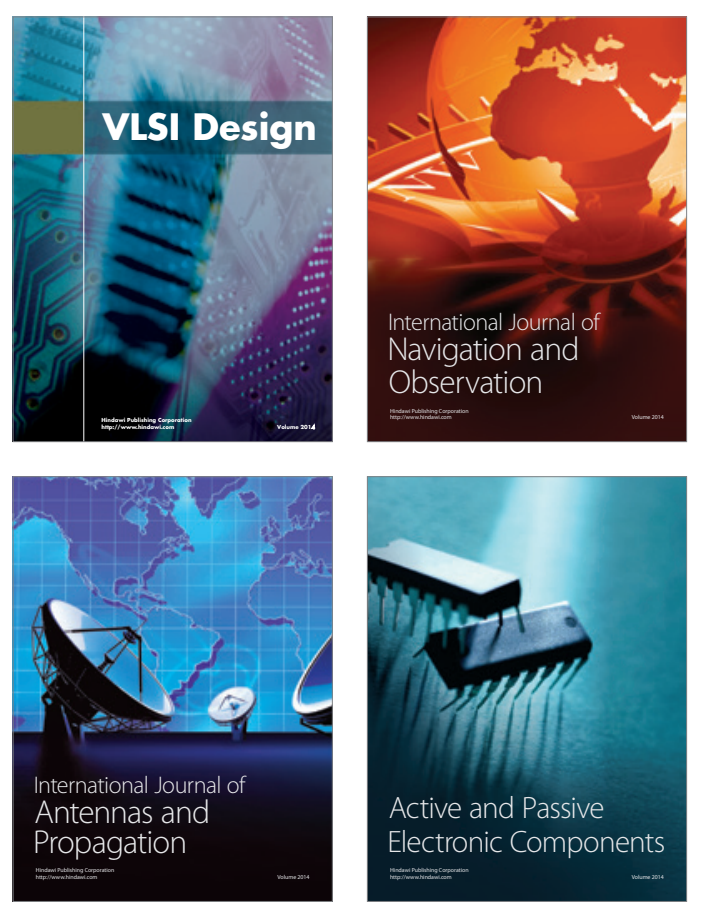
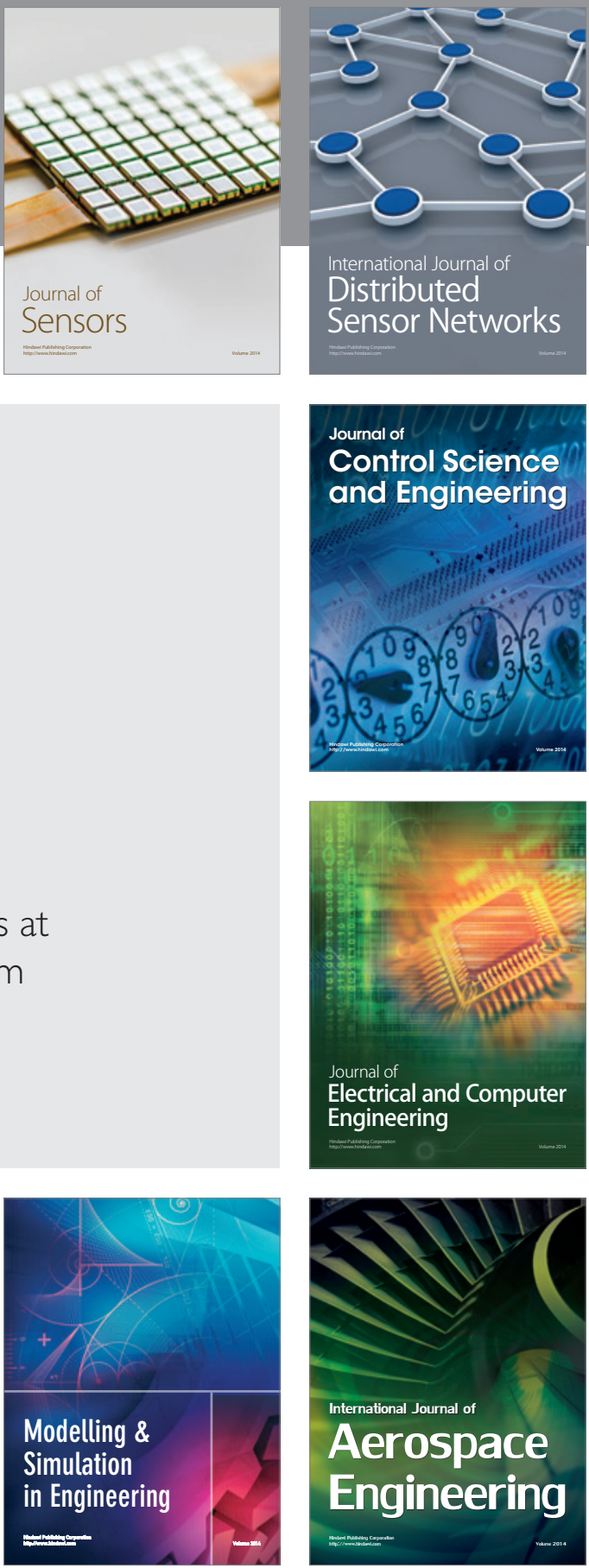

Journal of

Control Science

and Engineering
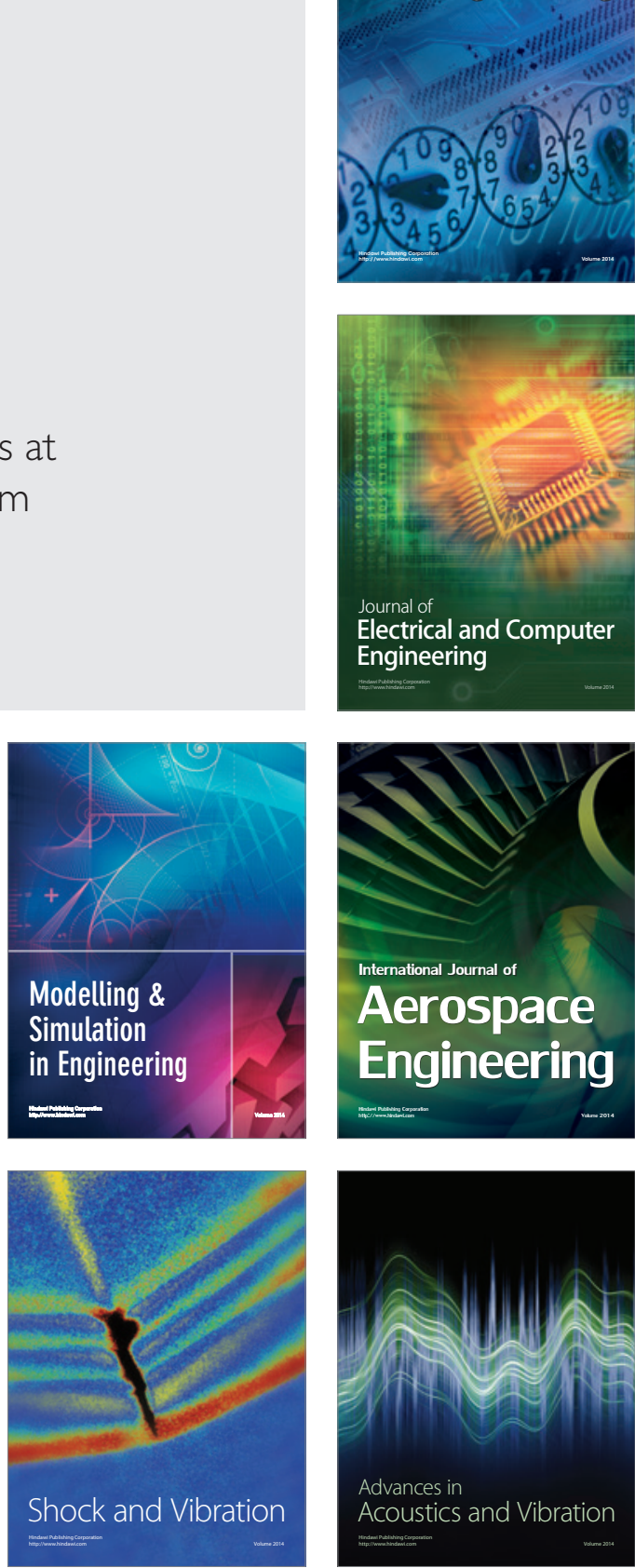